\title{
Analysis on the Causes of Mass Emergencies A Case Study of Weng'an Incident in Guizhou Province
}

\section{Yihao Shi}

\section{Department of Law and Politics, School of Humanities and Social Sciences, North China Electric} Power University, Baoding 071000, Hebei Province, China

\begin{abstract}
In the period of social transformation, the maintenance of social stability is the most basic requirement, and the outbreak of mass emergencies in recent years has brought great impact to the maintenance of social stability. Therefore, the reasons for exploring the mass emergencies are of great significance to the development of social stability. There are many reasons for the induction of group emergencies, and there are different conclusions in the analysis from different dimensions. Analysis on the reason of the multi-agent based on the case of Weng'an incident in Guizhou is the direction of the study. Exploring mitigation or solutions from this perspective provides basic information to effectively maintain social stability.
\end{abstract}

Keywords: Group emergencies; Causes; Guizhou Weng'an incident; Social stability; Multi-Agent analysis

\section{群体性突发事件的成因分析 \\ 一以贵州瓦安事件为例}

\author{
史益豪 \\ (华北电力大学人文与社会科学学院, 法政系, 河北 保定 071000 )
}

摘要: 在我国社会转型的攻坚期, 维护社会稳定是当前最基本的要求, 而近几年来不断爆发的群体性突发事件则给社会 稳定的维护带来了较大的冲击。因而, 探究群体性突发事件的原因对社会稳定的发展具有重要的意义。诱导群体性突发事件 的原因是多方面的, 从不同的维度进行分析会有不同的结论, 在以贵州瓮安事件为例的基础上进行多主体的原因分析是文章 进行研究的方向, 为从这一角度探索缓解或者是解决方法的研究提供了基本的资料, 从而来有效的维护社会稳定。

关键词：群体性突发事件；成因；贵州瓮安事件；社会稳定；多主体原因分析

引言

目前，我国处于社会转型的非常时期，促进社会矛盾的因素不断增加，群体性突发事件是指在短时间 内爆发的, 不同主体之间在涉及到切身利益的情况下, 采取静坐, 游行, 集会等方式来示威党政机关, 破 坏公共财物, 威胁公民人身安全并扰乱社会秩序的不良社会行为。近年来, 群体性突发事件的产生较为频 繁, 如果没有妥善处置, 那么群体性突发事件就会演变成性质更为恶劣的社会危机事件。2008 年发生的贵 州瓮安事件是一个产生了较为严重影响的事件, 以该事件为研究的主体, 对该群体性突发事件中的不同主 体进行论述, 力求为以后的研究达到举一反三的效果。

\section{1 群体性突发事件的基本特征}

我国发生了很多大大小小的群体性突发事件，要想对群体性突发事件有一个较为基本的认识，就得对 群体性突发事件的基本特征有一定的了解, 并且随着经济社会的发展, 群体性突发事件也会出现一些新的 
特征，把握其基本特征有助于我们更深入的了解群体性突发事件。

\section{1 突发事件的情绪性}

基本上发生的所有群体性突发事件都带有民众的情绪性, 贵州瓮安事件就有很强的代表性, 槩安事件 的导火索是女中学生的死因, 死者家属因不满政府给出的死亡证明以及政府相关人员恶劣的态度便开展了 一系列的社会活动, 在他们激烈的情绪化带动下以及政府留下的固有的负面印象, 社会上的大批人员便迅 速聚集到了一起, 对政府开展了持续七个小时的打砸抢烧的活动, 在事后的调查发现, 有相当一部分的闹 事人员是不清楚缘由的, 纯粹就是被其他人对政府的情绪化表露所带动的, 由此可见, 群体性突发事件的 情绪化是一个影响民众理智的重要因素, 如若拿捏不好便会成为别有用心的人的工具。

\section{2 突发事件的突发性}

群体性突发事件的爆发并不是循序渐进的, 而是发生的很突然, 它是人们的不满积累到一个程度后突 然的爆发, 并且冲击力较强, 影响力度较大, 一般来说在群体性事件出现之前, 就有一个问题积累的过程, 并且前兆也比较明显, 群体性突发事件产生的最大原因就是因为很多问题堆积到一起不能够解决或者是对 上级封锁了消息, 贵州㸗安事件的发生也不是偶然的, 群众们的爆发也是因为多年积累的不满找到了一个 宣泄的机会, 在瓮安, 盗窃, 抢劫, 杀人等刑事案件频频发生, 可是公安破案的几率却不到 $50 \%$, 这就引 起了人们的强烈不满, 并且大大的降低了对政府的信服度。因而对待民众的反映的每一个问题, 政府都要 认真及时的进行解决, 以免发生成为大规模的群体性突发事件。

\section{3 突发事件数量多, 规模大}

在 1993 年到 2003 年这十年间, 群体性突发事件的数量则由原来的 0.87 万起增加到了六万起, 十年 间增长了六倍多, 而且从 2007 年以来每年发生的群体性突发事件都在 9 万起以上, 更为严重的是特大群 体性突发事件的频率增加, 2008 年除了震惊中国的瓮安事件的发生, 还发生了很多大型的群体性突发事件, 有云南的孟连事件, 湖南的吉首事件, 甘肃的陇南事件等, 可见, 群体性突发事件的频率在不断的上升, 群体性突发事件的规模也在不断的扩大, 并且从参与的人数上来看, 1993 年到 2003 年这十年间参与的人 数由 37 万多人增加到了 307 万多人, 年均增长率达到了 $12 \%$, 并且群体性突发事件的参与人数百人以上者 由 1400 起增加到了 7000 多起, 可见群体性突发事件有着向恶性事件发展的趋势, 我们需要产生高度的警 觉。

\section{4 突发事件的时代性}

如今是一个互联网的时代, 社会上的任何风吹草动都会在网上激起不小的波澜, 在网上, 人们可以随 意的发表自己的言论, 并对自己所说的话不会负有相应的责任, 这种看似自由平等的互联网世界其实对任 何一个事件有着相当大的影响力, 尤其对群体性事件的影响更为深刻, 在群体性突发事件爆发后, 人们会 急切的想知道事件发生的起因和结果, 而上传信息的人难免会带有自己的主观色彩, 这样就会有失偏颇, 那么网上的與论就会对事件的不良发展起到了一个推波助澜的作用, 并且传播者自身辨别真伪的能力存在 着差异, 因而在传播信息的时候谣言也就随之产生了, 因而, 在这个互联网的时代, 网络对群体性突发事 件有着很大的影响, 群体性突发事件在掺杂着互联网这个时代所具有的特点后, 它波及的范围较广, 参与 的人数就会越来越多, 正确的利用互联网这个双刃剑对群体性突发事件的发展会产生较大的积极影响。 


\section{2 群体性突发事件的原因}

群体性突发事件不是一个简单的反映民意的事件，它的存在对百姓的生活带来了很大的威胁，更为严 重的还会影响到我国经济和政治的稳定发展, 这对我国建设和谐社会产生了不良的影响, 因而, 弄清楚群 体性突发事件的原因是进行预防和遏制的基础, 只有这样才能有效的构建和谐社会。

\section{1 民众方面}

\subsection{1 认识上较为偏颇}

在客观层面上, 人们对于党宣传的以人为本的科学发展观和立党为公, 执政为民的执政理念是表示支 持的, 但是他们并没有意识到要达到这些口号所宣传的理想状态是一个长期而且艰巨的任务, 在个人利益 和国家利益发生冲突的情况下, 人们通常关注的是个人利益, 可以说百姓的责任感与使命感并没有想象的 那么伟大, 因而, 当与自己切身相关的问题在没有得到解决的情况下, 人们通常就会采取较为过激的行为 来表达自己的需求。在群体性突发事件中, 人们通常都会带着盲目性。

在群体中，人们会很容易的受到当事人情绪的感染，在瓮安事件中，大部分闹事的人员都是受到别人 情绪的感染而加入闹事的队伍中, 这种盲目的加入带来的后果就是大批人员事后被公安拘留或者是关押, 他们为此付出了较为沉痛的代价, 而且在我国他们都有着 “大闹大解决, 小闹小解决, 不闹不解决” 的心 态, 所以在群体性突发事件中，人们一般都会有将事情闹大的想法和冲动。

\subsection{2 缺乏健全的法律知识}

群体性突发事件的发生从某个程度上可以表明人们的民主意识在不断的提高，人们懂得利用一定的方 式来维护自身的权利, 但是由于法律意识的淡薄导致了他们采取的方式不妥当, 并且容易受到别人的误导, 而我国的现状就是对法律的宣传力度不够大, 人们没有意识到掌握法律意识的重要性, 在他们的观念里, 法律是离他们很遥远的一个知识, 尤其是在农村和边远的地区, 法律还不如他们的祖训和家规来的有威信 和信服度。并且在瓮安事件中, 有大部分的青年纯粹是出于 “看热闹不嫌事大” 的态度加入了这个活动, 完全没有意识到这是违法活动, 以至于在事情结束后他们也为此付出了代价。可见, 进行普法教育是当务 之急。

黑恶势力以及别有用心之人的煽动

近年来, 黑恶势力在地方得到了较大的发展, 这些黑恶势力以及别有用心之人便会利用社会转型时期 人们的内部矛盾来进行恶意的宣传, 破坏社会的稳定, 更为严重的就是当地政府和黑恶势力进行勾结, 一 起做着损害百姓的事情。可以说瓮安事件就是一个掺杂着黑恶势力的事件, 这些地方黑恶势力利用政府工 作人员在相关工作中的不足来煽动群众进行打、砸、抢、烧等活动, 让地方政府付出了昂贵的代价。并且 据槩安县原公安局局长坦白, 警方内部人员有与黑恶势力进行勾结的。可见, 重塑政党之风以及打压地方 黑恶势力是缓解群体性突发事件的重要举措。

\section{2 政府方面}

\section{2.1 基层组织控制力减弱, 权威下降}

长期以来，基层组织对民众所反映的问题没有进行有效的调节或者是解决，人们对基层组织的信服度 大大的降低了, 因而基层组织对人们的号召力, 凝聚力的作用也减弱了, 再加上地方政府领导对群众所关 注的问题知之甚少或者是不管不问, 导致了本该由地方政府领导解决的问题难以解决或者是无法解决。因 
而, 在这样的情况下, 人们便将目光转移到了国家的层面上, 群众在遇到自身问题无法解决的前提下, 便 会想办法得到国家的注意, 而 “法不责众” 的观念早已深入人心, 因而单个的社会成员就意识到了群体性 活动的重要性。就㸗安事件来说, 死者家属进行游行, 示威等活动就是为了能得到国家的关注, 他们对当 地政府进行的一系列活动都表明了对政府的失望, 要求政府能在国家的压力下给予他们答复, 可见, 群众 进行群体性事件在很大程度上都是因为当地政府的不作为, 他们希望通过一个激进的方式来促进政府的改 进。

\section{2 .2 贪污腐败之风盛行, 干群矛盾严重}

基层干部是最贴近群众的，他们应该做到及时反映群众需求以及要解决的问题，可是在现实情况中， 基层干部对群众的呼声和困难却视而不见, 在贪污腐败盛行的年代, 很少有干部还能做到洁身自好的, 他 们热衷于随波逐流, 和群众之间的距离越来越远, 他们有的追求享乐主义, 只关注自身的利益, 有着强烈 的拜金主义; 而有的干部却为了追求政绩, 开展形象工程和面子工程, 甚至是不惜以损害百姓的利益为前 提; 有的干部更为直接, 他们假公济私, 奢侈浪费……所有这些都说明了干部都只是站在自己的角度来做 事, 他们不仅不能解决群众需要解决的问题, 还以权谋私, 做出一些损害群众利益的事情, 使得干群矛盾 更加的紧张, 而在群众根深蒂固的思想里, 干部是既得利益着, 他们应该为群众多办事, 积极的听取群众 的意见, 然而现实却和群众的理想状态是截然相反的, 在这种极大的反差下, 激起了群众极大的不满, 最 终导致了冲突。瓮安事件就是一个典型的例子, 正是由于瓮安县干部的不作为, 让群众心里聚集起了一定 程度的怨恨和不满, 因而在导火索的牵引下便发生了这起震惊全国的群体性突发事件。

\subsection{3 政府人员在职能上的缺位和越位}

政府人员在面对群体性突发事件应该牢牢的记住自己的职能, 不能有越位、错位和缺位的现象, 不然 就会将矛盾进一步的激化。瓮安事件的产生并不是偶然的, 而是民众长期积累的不满所造成的, 这就是因 为政府人员工作的不到位, 在女中学生死亡的这个导火索中, 政府部门的人员还和死者家属产生了激烈的 肢体冲突, 这就是政府人员在逃避责任的表现, 这也是造成矛盾激化的最主要方面, 若政府相关人员能将 矛盾在萌芽时期予以解决, 积极的对待民众的诉求, 那么事情的严重性就不会到如此恶劣的地步, 他们将 简单的人事纠纷上升到了民众和政府之间的矛盾纠纷, 这就是矛盾升级的表现。因而政府人员应该牢牢的 记住自己的职能, 不能缺位、越位和错位。对待民众的诉求要及时的解决, 达到让民众满意的效果。

\section{3 客观层面}

\subsection{1 民众表达诉求的渠道不畅通}

群众闹事, 那是基于自己的问题长久得不到政府的解决从而产生了不满, 长期积累后找到了一个突破 口便会宣泄出来, 瓮安事件就是如此。在瓮安, 社会治安特别的差, 人们生活在一个没有安全感的环境里, 群众不断的向政府反映此事, 但结果还是杳无音信, 可以说诉求渠道的不畅通, 人们的问题得不到解决就 会演化成为性质较为恶劣的群众闹事事件, 因而, 需要建立一个和群众有着良好互动的平台, 能让群众反 映的问题得到相关部门的了解, 也能让群众知道相关部门的态度或者是措施, 这种双向直接互动的平台能 够在很大程度上减少民众的不满情绪。

\section{3.2 没有完善的民意纠纷调解机制}

民意纠纷的调解本来是可以在萌芽状态得到遏制的, 但是由于缺乏有效的调解机制, 导致了矛盾的不 
断升级, 最终发展成为群体性突发事件, 对社会的和谐发展造成了极大的威胁。解决群体性突发事件最为 有效的办法是从源头上进行遏制, 要建立一个完善的调解纠纷的体制, 对民众反映的问题力争做到早报告, 早解决。要充分发挥基层干部和群众直接互动的优势, 将民众的涉及到的各类问题争取在萌芽状态下予以 解决。

\section{3 结语}

群体性突发事件大规模的发生, 反映了我国地方治安严重的不足, 尤其是地方领导和基层干部的不作 为, 无视民众的诉求更加激化了干群矛盾, 为社会和谐的发展埋下了一颗不定时的炸弹。在社会转型的攻 坚时期, 应该要全面落实以人为本的科学发展观, 建设好服务型的政府, 积极的缓解干群矛盾, 完善民众 诉求的机制，将矛盾扼杀在萌芽之中，从而为社会的稳定和健康发展奠定了基础。

\section{参考文献:}

[1] 胡红霞, 王虹. 群体性突发事件背后民众利益诉求困境与化解思路 [J]. 北京工业大学学报 (社会科学 版), 2010, 04:28-32.

[2]吴佩芬, 王国明. 近几年学术界关于群体性事件的研究综述 [J]. 云南行政学院学报, 2009, 05:45-48.

[3] 中国行政管理学会课题组. 我国转型期群体性突发事件主要特点、原因及政府对策研究 [J]. 中国行政管 理, 2002, 05:6-9.

[4]李一平. 突发性群体事件的成因及防范 [J]. 中州学刊, 2002, 05:170-174.

[5]刘华欣．群体性突发事件网络與情演变机制的传播学解读 [J]。新闻知识, 2013, 03:10-12.

[6]徐寅峰,刘德海．群体性突发事件产生根源的主观博亦分析 [J]．预测, 2004, 06:43-45+9.

[7]沙莲香著: 《社会心理学》, 中国人民大学出版社 2002 年版, 第 221-222 页。

[8]朱力: 《中国社会风险解析一群体性事件的社会冲突性质》，第 69-78 页。

[9]王国勒：《社会网络视野下的集体行动》，中国人民大学 2008 年博士论文，第 5 页。

[10] 邱泽奇: 《群体性事件与法治发展的社会基础》，《云南大学学报》 2004 年第 5 期，第 54-96 页。

\section{References:}

[1] Hu Hongxia, Wang Hong. People's Dilemma and Resolving Ideas of People's Interests Behind Group Emergencies [J]. Journal of Beijing University of Technology (Social Science Edition), 2010, 04: 28-32 (in Chinese with English abstract).

[2] Wu Peifen, Wang Guoming. Analysis of the research on group events in recent years [J]. Journal of Yunnan Administration College, 2009, 05: 45-48.

[3] China Administration Institute Task Force. the main features, the reasons and government countermeasures of the incident in China's transition period[J]. China Administration, 2002, 05: 6-9.

[4] Li Yiping. Causes and prevention of sudden group events [J]. Zhongzhou Journal, 2002, 05: 170-174.

[5] Liu Huaxin. Interpretation on the evolution mechanism of public opinion communication in the group of emergencies network [J]. News knowledge, 2013, 03: 10-12.

[6] Xu Yinfeng, Liu Dehai. Subjective game analysis on the origin of group emergencies [J]. Forecast, 2004, 06: 43-45 + 9.

[7] Sha lianxiang. "Social Psychology", Renmin University of China Press, 2002 edition, pages 221-222. 
[8] Zhu Li: "China's social risk analysis - the social conflict of mass incidents," pages 69-78.

[9] Wang Guole: "social network under the vision of the collective action", the Chinese People's University 2008 Doctoral Thesis, page 5.

[10] Qiu Zeqi: "Mass incidents and the social basis on the development of the rule of law", "Journal of Yunnan University" 2004, the Fifth Period, pages 54-96. 